\title{
NGHIÊN CÚU TĂNG GLUCOSE MÁU DO STRESS TRONG TIÊN LƯợNG NHỒI MÁU CƠ TIM CẤP KHÔNG ĐÁI THÁO ĐƯờNG
}

\author{
Mai Thiện Nhân ${ }^{1}$, Hoàng Anh Tiến ${ }^{2}$, Hồ Anh Bình ${ }^{1}$ \\ 1. Bệnh viện Trung uoong Huế \\ 2.Truờng Đại học Y Dược Huế
}

DOI: $10.47122 / v j d e .2020 .38 .6$

\section{ABSTRACT \\ Stress hyperglycemia and prognosis of acute myocardial infarction in nondiabetic patients: a cross-sectional study}

Background: Acute hyperglycemia on admission is common in acute myocardial infarction(AMI)patients regardless of diabetic status, and is known as one of prognostic factors. However there is limited evidence on the effect of acute hyperglycemia on nondiabetic patients. Our goal is to assess if admission hyperglycemia increases the risk for in-hospital adverse clinical events and mortality after AMI in non-diabetic patients. Methods: We perform this cross-sectional study involving 128 AMI patients admitted to the Cardiovascular Emergency and Interventional Department at Hue Central Hospital between April 2017 and July 2018. We categorize patients according to their glucose levels at admission $(<7,8 ; 7,8-11,0$ and $\geq 11,1 \mathrm{mmol} / \mathrm{L})$ and compare in-hospitaladverse clinical events andmortality across these admission glucose categories. We employ logistic regression to assess the differences in outcomes across admission glucose levels while adjusting for the same covariates. Results: As compared to patients with admission glucose $<7,8 \mathrm{mmol} / \mathrm{L}$ $(70,3 \%)$, patients with admission glucose 7,8$11,0 \mathrm{mmol} / \mathrm{L}(23,4 \%$, odds ratio $O R=2,09$; 95\%confidence interval $C I[0,65-6,77])$ and $>11,1 \mathrm{mmol} / \mathrm{L}(6,3 \%$, OR $=7,56 ; 95 \% \mathrm{CI}$ $[1,29-44,28])$ are at elevated in-hospital adverse clinical events after adjustment for patients' demographic characteristics and clinical status. Among non-diabetic patients with admission glucose 7,8-11,0 mmol/L (OR $=1,46 ; 95 \%$ CI $[0,34-6,37])$ and $>11,1$ $\mathrm{mmol} / \mathrm{L}(O R=7,82 ; 95 \% C I[1,39-43,90])$ remain elevated risk for adjusted in-hospital mortality.In the multivariate analysis, an increment of $1 \mathrm{mmol} / \mathrm{L}$ in blood glucose on admission is associated with an increase in inhospital adverse clinical events andmortality risk of $41 \%$ and 26\%, respectively. Conclusions: These findings underscore clinical significance of admission hyperglycemia on in-hospital adverse clinical eventsand mortality in non-diabetic AMI patients.

Key words: Acute Myocardial Infarction; Admission glucose;Non-diabetic; Adverse clinical events; Mortality.

\section{TÓM TẮT}

Tổng quan: Tăng glucose máu cấp lúc nhập viện xảy ra phổ biến ở bệnh nhân nhồi máu cơ tim (NMCT) cấp bất kể có đái tháo đường (ĐTĐ) hay không, và được biết đến như là một trong các yếu tố tiên lượng. Tuy nhiên, có rất ít bằng chứng về ảnh hưởng của tăng glucose máu cấp ở bệnh nhân không có ĐТÐ. Mục tiêu nghiên cúu của chúng tôi: Là đánh giá xem thử tăng glucose máu lúc nhập viện có làm tăng các nguy cơ biến chứng tim mạch và tử vong trong viện sau NMCT cấp ở bệnh nhân không ĐTĐ hay không. Phương pháp: Là nghiên cứu mô tả cắt ngang, gồm 128 bệnh nhân $\mathrm{NMCT}$ cấp nhập viện khoa Cấp cứu Tim mạch Can thiệp, Bệnh viện Trung ương Huế từ tháng 4/2017 đến $7 / 2018$. Các bệnh nhân nay được chia thành 3 nhóm glucose theo glucose máu lúc nhập viện $(<7,8 ; 7,8-11,0$ và $\geq 11,1 \mathrm{mmol} / \mathrm{L})$ và so sánh các biến chứng tim mạch và tử vong xảy ra trong viện theo các nhóm glucose máu nhập viện. Hồi qui logistic được sử dụng để đánh giá sự khác biệt về kết cục lâm sàng giữa các nhóm glucose máu nhập viện sau khi điều chỉnh các biến nhiễu. Kết quả: Khi so 
sánh với nhóm bệnh nhân có glucose nhập viện $<7,8 \mathrm{mmol} / \mathrm{L}$ (70,3\%), những bệnh nhân có glucose $7,8-11,0 \mathrm{mmol} / \mathrm{L}(23,4 \%$, tỉ số odds $\mathrm{OR}=2,09$; độ tin cậy $95 \% \mathrm{CI}[0,65$ $6,77])$ và $>11,1 \mathrm{mmol} / \mathrm{L}(6,3 \%, \mathrm{OR}=7,56$; 95\% CI [1,29-44,28]) tăng tỉ lệ các biến chứng tim mạch trong viện sau khi điều chỉnh với các yếu tố lâm sàng và nhân khẩu học của bệnh nhân. Những bệnh nhân không ĐTĐ với glucose máu nhập viện 7,8-11,0 $\mathrm{mmol} / \mathrm{L}$ $(\mathrm{OR}=1,46 ; 95 \% \mathrm{CI}[0,34-6,37])$ và $>11,1$ $\mathrm{mmol} / \mathrm{L} \quad(\mathrm{OR}=7,82 ; 95 \% \mathrm{CI}[1,39-43,90])$ cũng tăng nguy cơ tử vong trong viện. Trong phân tích đa biến, cứ tăng $1 \mathrm{mmol} / \mathrm{L}$ glucose nhập viện liên quan đến tăng tỉ lệ các biến chứng tim mạch và tử vong trong viện lần lượt là $41 \%$ và $26 \%$. Kết luận: Những kết quả này nhấn mạnh ý nghĩa lâm sàng của tăng glucose máu lúc nhập viện đối với các biến chứng tim mạch và tử vong trong viện ở bệnh nhân NMCT cấp không ĐTĐ.

Tù khóa: Nhồi máu co tim cấp; Glucose máu nhập viện; Không đái tháo đường; Các biến chứng tim mach; Tư vong.

Chịu trách nhiệm chính: Hồ Anh Bình

Ngày nhận bài: 11/01/2020

Ngày phản biện khoa học:12/01/2020

Ngày duyệt bài: 27/02/2020

Email: drhoanhbinh@gmail.com

ĐT: 0913489896

\section{1. ĐẠT VẤN ĐỀ}

Nhồi máu cơ tim là một bệnh lý cấp cứu nội khoa thường gặp, có nhiều biến chứng nguy hiểm và có tỉ lệ tử vong cao nếu không được phát hiện và xử trí kịp thời.

Tăng glucose máu lúc mới nhập viện là một biểu hiện thường gặp trong hội chứng vành cấp và nó liên quan đến một kết cục xấu ở cả bệnh nhân có đái đường và không đái đường [12].

Bất kể giá trị của điểm cắt là bao nhiêu thì tăng glucose máu do stress thường hay được định nghĩa là tình trạng tăng glucose máu tự mất đi sau khi hết tình trạng cấp tính của bệnh [6]. Do đó chúng tôi tiến hành nghiên cứu này với mục tiêu tìm hiểu mối liên quan của nồng độ glucose máu lúc nhập viện với các biến cố tim mạch xảy ra trong thời gian nằm viện.

\section{2. ĐỐI TƯợNG, PHƯƠNG PHÁP NGHIÊN CÚ'U}

\section{1. Đối tượng nghiên cứu}

Đối tượng nghiên cứu được chọn ra liên tiếp từ các bệnh nhân được chẩn đoán $\mathrm{NMCT}$ cấp nhập viện tại khoa Cấp cứu tim mạch can thiệp, Bệnh viện Trung ương Huế trong thời gian 4/2017-7/2018.

\section{Tiêu chuẩn chọn bệnh:}

- Bệnh nhân được chẩn đoán NMCT cấp dựa theo định nghĩa $\mathrm{NMCT}$ toàn cầu lần thứ tư [11].

- Bệnh nhân nhập viện tại khoa trong vòng $\leq 48$ giờ kể từ khi có triệu chứng.

- Glucose máu nhập viện được xét nghiệm trong vòng 24 giờ kể từ lúc vào viện.

Tiêu chuẩn loại trừ:

- Có ĐTĐ:

+ Được chẩn đoán ĐTĐ trong quá khứ.

+ ĐTĐ mới được chẩn đoán trong khi nằm viện (dựa vào glucose máu đói hoặcHbA1C theo tiêu chuẩn của ADA 2018 [3]).

- Không đồng ý tham gia nghiên cứu.

-Trước đó dùng các thuốc làm tăng glucose máu.

\subsection{Thiết kế nghiên cứu:}

Mô tả cắt ngang

\section{Chỉ tiêu lâm sàng}

Các chỉ tiêu lâm sàng được phân tích dựa vào glucose máu lúc nhập viện gồm:

- Các biến chứng tim mạch trong viện: shock tim, suy tim sung huyết, loạn nhịp và ngừng tim.

- Tử vong trong bệnh viện: gồm có trường hợp tử vong thật sự trong khi nằm viện và trường hợp bệnh nhân nặng, tiên lượng tử vong gần, được người nhà bệnh nhân xin về vì từ chối điều trị. 


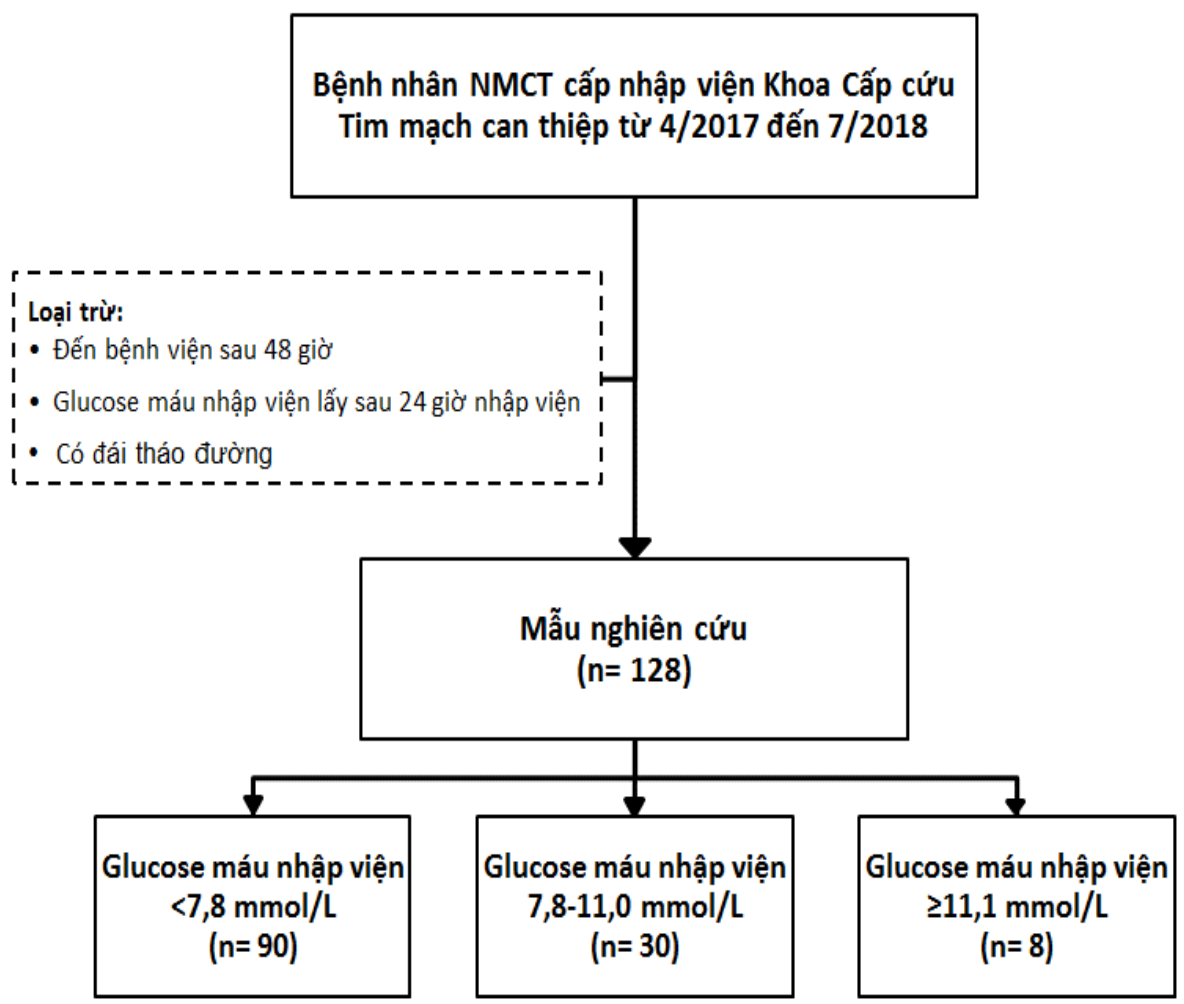

Hình 1.Sơ đồ chọn bệnh nhân

\subsection{Xử lý số liệu:}

- Sử dụng tỉ lệ phần trăm và số lượng để mô tả các biến phân loại. Sử dụng thích hợp trung vị và độ trải giữa (IQR) (có phân phối không chuẩn) hay trung bình và độ lệch chuẩn (có phân phối chuẩn) để mô tả các biến liên tục.

- Sử dụng test Chi bình phương (cho các tỉ lệ) hoặc test Kruskall-Wallis/Mann Whitney U (cho các giá trị trung vị) để đánh giá sự khác biệt về mặt thống kê giữa các nhóm glucose máu.

- Hồi qui logistic đa biến được sử dụng để đánh giá xem mối liên quan giữa glucose máu nhập viện và tỉ lệ tử vong/các biến chứng của
NMCT xảy ra trong bệnh viện.

- Phân tích hồi qui Cox đa biến để đánh giá xem mối liên quan giữa glucose máu nhập viện và tỉ lệ tử vong trong bệnh viện theo thời gian.

- Các kết quả của các mô hình phân tích thống kê được thể hiện bằng tỉ số odds (OR), tỉ số rủi ro (HR), khoảng tin cậy $95 \%(95 \%$ $\mathrm{CI})$. Tất cả các so sánh có kèm theo giá trị $\mathrm{p}$, với $\mathrm{p}<0,05$ được xem là sự khác biệt có ý nghĩa thống kê.

- Tất cả các phân tích thống kê được thực hiện bằng phần mềm SPSS (Statistical Package for Social Science) cho hệ điều hành Windows, phiên bản 23.0. 


\section{KẾT QUẢ VÀ BÀN LUẬN}

\subsection{Phân bố nồng độ glucose máu nhập viện}

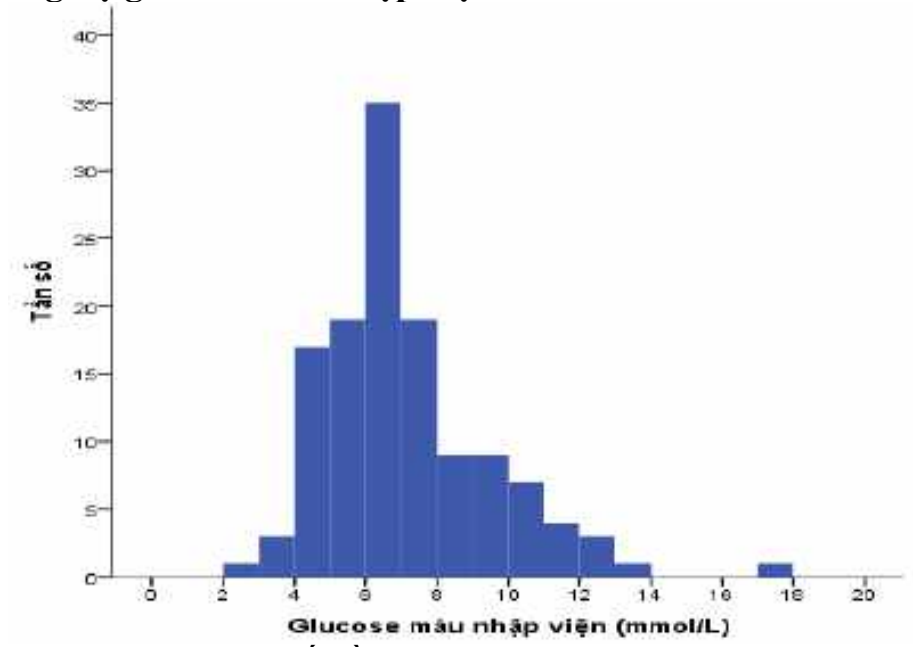

Hình 2. Phân bố nồng độ glucose máu lúc nhập viện

Bảng 1. Các nhóm glucose máu lúc nhập viện

\begin{tabular}{|c|c|c|}
\hline Glucose máu nhập viện (mmol/L) & Số bệnh nhân (n) & Phần trăm (\%) \\
\hline$<7,8$ & 90 & 70,3 \\
\hline $7,8-11,0$ & 30 & 23,4 \\
\hline$\geq 11,1$ & 8 & 6,3 \\
\hline Tổng & 128 & 100,0 \\
\hline
\end{tabular}

Giá trị trung vị glucose máu lúc nhập viện là 6,6 (IQR 5,5-8,0) mmol/L. Trung vị giá trị glucose của các bệnh nhân trong mẫu nghiên của chúng tôi cũng gần tương tự nghiên cứu của nhóm tác giả Shi Zhao và cộng sự năm 2017 trên 8258 bệnh nhân không có tiền sử ĐTĐ tại các bệnh viện ở Trung Quốc cho kết quả là 6,4 (IQR 5,3-8,0) mmol/L [11]. Đa số bệnh nhân tập trung ở nhóm có glucose máu lúc nhập viện $<7,8$ mmol/L (chiếm 70,3\%). Nhóm có glucose máu vừa và cao $(\geq 7,8 \mathrm{mmol} / \mathrm{L})$ chiếm $29,7 \%$.

\section{2. Đặc điểm các biến cố tim mạch trong viện}

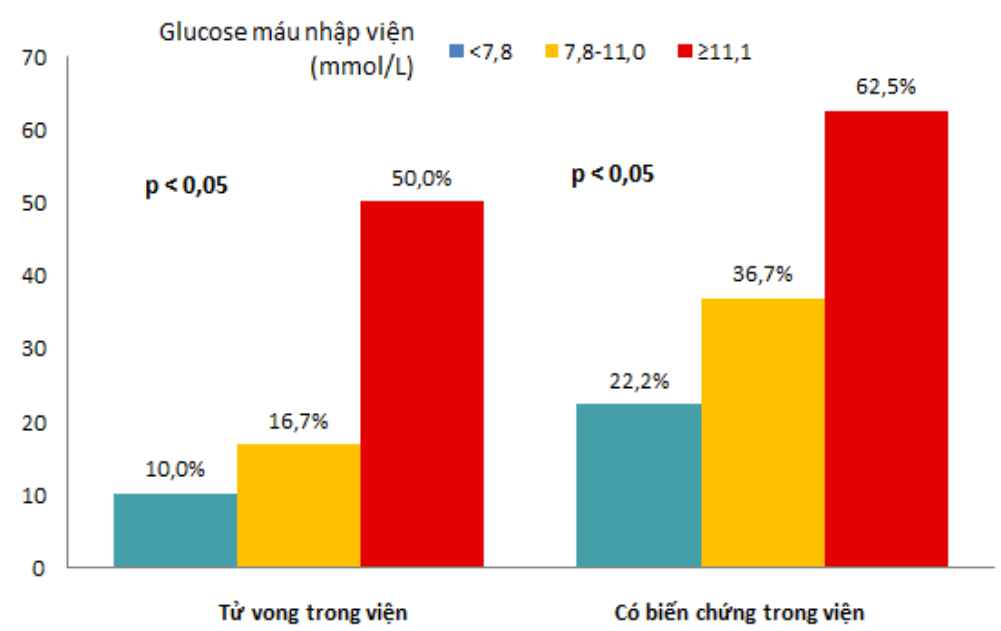

Hình 3. Biến chứng tim mạch và tử vong trong viện giữa các nhóm glucose nhập viện 
Tỉ lệ tử vong chiếm 14,1\% tổng số bệnh nhân. Và nhóm có glucose máu cao có tỉ lệ phần trăm cao hơn nhiều so với hai nhóm kia. Kết quả nghiên cứu về mối liên quan giữa glucose máu nhập viện và tỉ lệ tử vong trong viện gần như tương tự các kết quả của các nghiên cứu khác ở nhiều nhóm bệnh nhân thuộc các chủng tộc khác nhau. Nghiên cứu của Nguyễn Văn Tân năm 2015 cho thấy tỉ lệ tử vong trong thời gian nằm viện bao gồm các trường hợp tử vong và bệnh nặng xin về là $12 \%$ [2]. Có $28,1 \%$ bệnh nhân có ít nhất một biến chứng tim mạch xảy ra trong viện. Tương tự tỉ lệ tử vong, nhóm có nồng độ glucose máu càng cao thì tỉ lệ xảy ra biến chứng càng nhiều. Nghiên cứu của Pei-Chi Chen cho thấy tỉ lệ các biến cố xảy ra trong viện ở nhóm có glucose máu $\geq 13,89 \mathrm{mmol} / \mathrm{L}$ là $48,7 \%$ [4].

Bảng 2. Đặc điểm biến chứng tim mạch trong viện theo nhóm glucose nhập viện

\begin{tabular}{|c|c|c|c|c|c|c|}
\hline \multirow{2}{*}{\multicolumn{2}{|c|}{ Đặc điểm }} & \multirow{4}{*}{$\begin{array}{c}\begin{array}{c}\text { Chung } \\
(\mathbf{n}=\mathbf{1 2 8})\end{array} \\
15 \\
11,7\end{array}$} & \multicolumn{3}{|c|}{ Glucose nhập viện (mmol/L) } & \multirow{4}{*}{$\begin{array}{c}\mathbf{p} \\
>0,05\end{array}$} \\
\hline & & & \multirow{2}{*}{$\begin{array}{c}\begin{array}{c}<7,8 \\
(\mathbf{n}=90)\end{array} \\
7\end{array}$} & \multirow{2}{*}{$\begin{array}{c}\begin{array}{c}\mathbf{7 , 8 - 1 1 , 0} \\
(\mathbf{n}=\mathbf{3 0})\end{array} \\
6\end{array}$} & \multirow{2}{*}{ 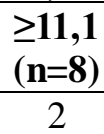 } & \\
\hline Suy tim & $\mathrm{n}$ & & & & & \\
\hline sung huyết & $\%$ & & 7,8 & 20,0 & 25,0 & \\
\hline \multirow{2}{*}{ Sốc tim } & $\mathrm{n}$ & 17 & 9 & 6 & 2 & \multirow{2}{*}{$>0,05$} \\
\hline & $\%$ & 13,3 & 10,0 & 20,0 & 25,0 & \\
\hline \multirow{2}{*}{ Loạn nhịp } & $\mathrm{n}$ & 10 & 7 & 2 & 1 & \multirow{2}{*}{$>0,05$} \\
\hline & $\%$ & 7,8 & 7,8 & 6,7 & 12,5 & \\
\hline \multirow{2}{*}{ Ngừng tim } & $\mathrm{n}$ & 14 & 8 & 3 & 3 & \multirow{2}{*}{$<0,05$} \\
\hline & $\%$ & 10,9 & 8,9 & 10,0 & 37,5 & \\
\hline
\end{tabular}

Suy tim sung huyết trong thời gian nằm viện chiếm $11,7 \%$ số bệnh nhân. Nhóm có glucose máu cao và vừa $(45,0 \%)$ có tỉ lệ suy tim sung huyết cao hơn nhóm còn lại $(6,7 \%)$. Quan sát của chúng tôi tương tự kết quả của các nghiên cứu khác.

Nguyễn Văn Chi (2011) nghiên cứu cho thấy nhóm bệnh nhân có glucose máu cao $\geq 11,1 \mathrm{mmol} / \mathrm{L}$ có tỉ lệ bệnh nhân suy tim sung huyết là $57,0 \%$ [1].

Sốc tim chiếm 13,3\% bệnh nhân. Tương tự, nhóm có glucose máu cao và vừa có tỉ lệ này cao hơn nhóm còn lại. Nhóm có glucose

máu $\geq 11,1 \mathrm{mmol} / \mathrm{L}$ chiếm tỉ lệ $25 \%$. Các nghiên cứu sau cũng cho kết quả tương tự như Nguyễn Văn Chi (2011), nhóm bệnh nhân có glucose máu cao $\geq 11,1 \mathrm{mmol} / \mathrm{L}$ có tỉ lệ bệnh nhân sốc tim là $24,7 \%$ [1].

Có $10,9 \%$ bệnh nhân ngừng tim trong thời gian nằm viện. Nhóm có glucose máu cao ( $\geq 11,1 \mathrm{mmol} / \mathrm{L})$ có tỉ lệ bệnh nhân ngừng tim cao hơn khoảng 4 lần so với hai nhóm còn lại. Nghiên cứu của Ahmet E. (2013), tî lệ bệnh nhân có rung thất/nhanh thất ở nhóm có glucose máu cao là $18,1 \%$ trong khi nhóm glucose máu thấp chỉ là $3 \%$ [7].

\subsection{Liên quan giữa glucose máu nhập viện và các biến chứng tim mạch trong bệnh viện}

Bảng 3. Các biến chứng trong viện theo các nhóm glucose nhập viện

\begin{tabular}{|c|c|c|c|c|c|c|c|c|}
\hline \multirow{2}{*}{$\begin{array}{c}\text { Glucose } \\
\text { nhập viện } \\
(\mathbf{m m o l} / \mathbf{L})\end{array}$} & \multicolumn{2}{|c|}{$\begin{array}{c}\text { Biến chứng } \\
\text { trong viện }\end{array}$} & \multicolumn{3}{|c|}{ Trước điều chỉnh } & \multicolumn{3}{c|}{ Sau điều chỉnh } \\
\cline { 2 - 9 } & $\mathbf{n}$ & $\mathbf{\%}$ & $\mathbf{O R}$ & $\mathbf{9 5 \%} \mathbf{C I}$ & $\mathbf{p}$ & $\mathbf{O R}$ & $\mathbf{9 5 \%}$ CI & $\mathbf{p}$ \\
\hline$<7,8$ & 20 & 22,2 & 1 & - & - & 1 & - & - \\
\hline $7,8-11,0$ & 11 & 36,7 & 2,03 & $0,83-4,95$ & $>0,05$ & 2,09 & $0,65-6,77$ & $>0,05$ \\
\hline$\geq 11,1$ & 5 & 62,5 & 5,83 & $1,28-26,54$ & $<\mathbf{0 , 0 5}$ & 7,56 & $1,29-44,28$ & $<\mathbf{0 , 0 5}$ \\
\hline Chung & 36 & 28,1 & 1,35 & $1,13-1,62$ & $<\mathbf{0 , 0 5}$ & 1,41 & $1,13-1,76$ & $<\mathbf{0 , 0 5}$ \\
\hline
\end{tabular}


Trước điều chỉnh, so với nhóm glucose $<7,8 \mathrm{mmol} / \mathrm{L}$, nhóm glucose máu vừa có odds biến chứng tim mạch cao hơn 2,03 lần nhưng không có ý nghĩa thống kê. Nhóm glucose máu cao có odds biến chứng cao gấp 5,83 lần và khác biệt có ý nghĩa thống kê. Sau điều chỉnh cho tuổi, giới, nhịp tim, STEMI/NSTEMI và $\mathrm{EF}$, so với nhóm glucose máu $<7,8 \mathrm{mmol} / \mathrm{L}$, nhóm glucose máu vừa có odds biến chứng cao hơn 2,09 lần nhưng không có ý nghĩa thống kê. Nhóm glucose máu cao có odds biến chứng cao gấp 7,56 lần và có ý nghĩa thống kê.

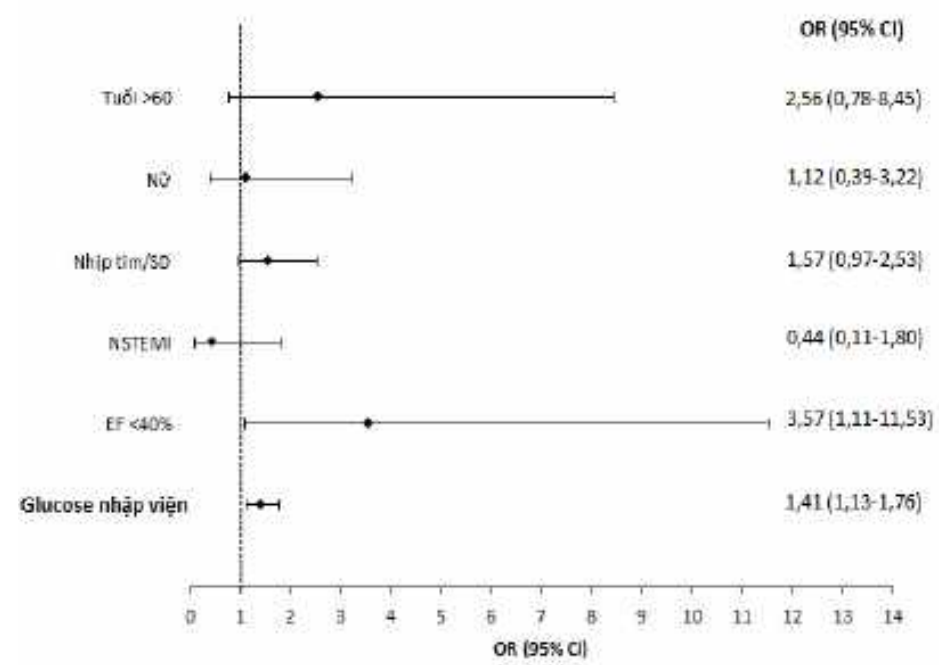

Hình 4. Ảnh hưởng của các biến tiên lượng lên biến chứng tim mạch trong viện

Sau điều chỉnh, glucose máu nhập viện vẫn có mối liên quan với biến chứng tim mạch trong viện, độc lập với các biến nhiễu. Cứ tăng mỗi $1 \mathrm{mmol} / \mathrm{L}$ glucose làm tăng odds biến chứng 1,41 lần hay $41 \%$ (có ý nghĩa thống kê).

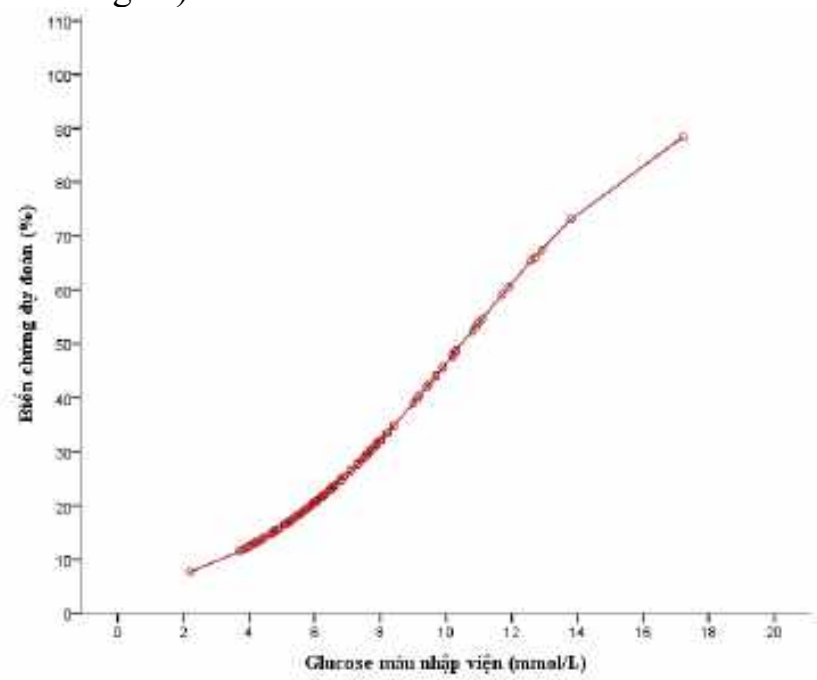

Hình 5. Xác suất dự đoán biến chứng tim mạch trong viện theo glucose máu nhập viện qua mô hình hồi qui logistic

Mô hình dự đoán xác suất có biến chứng dựa vào glucose nhập viện có hàm số $p(x)=$ $\frac{\exp (0,301 * x-3,157)}{1+\exp (0,301 * x-3,157)}$, ta thấy rằng glucose máu lúc nhập viện càng cao thì xác suất xảy ra các biến chứng tim mạch càng cao. 


\subsection{Liên quan giữa glucose máu nhập viện và tử vong trong bệnh viện}

Bảng 4. Tử vong trong viện theo nhóm glucose nhập viện

\begin{tabular}{|c|c|c|c|c|c|c|c|c|}
\hline \multirow{2}{*}{$\begin{array}{c}\text { Glucose } \\
\text { nhập viện } \\
(\mathbf{m m o l} / \mathbf{L})\end{array}$} & \multicolumn{2}{|c|}{$\begin{array}{c}\text { Tử vong } \\
\text { trong viện }\end{array}$} & \multicolumn{3}{|c|}{ Trước điều chỉnh } & \multicolumn{3}{c|}{ Sau điều chỉnh } \\
\cline { 2 - 9 } & $\mathbf{n}$ & $\mathbf{\%}$ & $\mathbf{O R}$ & $\mathbf{9 5 \%}$ CI & $\mathbf{p}$ & $\mathbf{O R}$ & $\mathbf{9 5 \%}$ CI & $\mathbf{p}$ \\
\hline$<7,8$ & 9 & 10,0 & 1 & - & - & 1 & - & - \\
\hline $7,8-11,0$ & 5 & 16,7 & 1,80 & $0,55-5,87$ & $>0,05$ & 1,46 & $0,34-6,37$ & $>0,05$ \\
\hline$\geq 11,1$ & 4 & 50,0 & 9,00 & $1,92-42,30$ & $<\mathbf{0 , 0 5}$ & 7,82 & $1,39-43,90$ & $<\mathbf{0 , 0 5}$ \\
\hline Chung & 18 & 14,1 & 1,31 & $1,08-1,59$ & $<\mathbf{0 , 0 5}$ & 1,26 & $1,01-1,57$ & $<\mathbf{0 , 0 5}$ \\
\hline
\end{tabular}

Trước điều chỉnh, glucose máu nhập viện tăng $1 \mathrm{mmol} / \mathrm{L}$ thì odds tử vong tăng lên $31 \%$ (có ý nghĩa thống kê). So với nhóm glucose máu $<7,8 \mathrm{mmol} / \mathrm{L}$, nhóm glucose máu vừa có odds tử vong cao hơn 1,8 lần nhưng không có ý nghĩa thống kê; nhóm glucose máu cao có odds tử vong cao gấp 9,0 lần và khác biệt có ý nghĩa thống kê. Sau điều chỉnh cho tuổi, giới tính, phân độ Killip, sốc tim thì các tỉ số odds của glucose máu với biến cố tử vong giảm so với trước. So với nhóm có glucose máu $<7,8$ $\mathrm{mmol} / \mathrm{L}$, nhóm có glucose máu vừa có odds tử vong giảm còn 1,46 nhưng không có ý nghĩa thống kê; nhóm glucose máu cao có odds tử vong giảm còn 7,82 và có ý nghĩa thống kê. Biến liên tục glucose máu vẫn có mối liên quan với tử vong trong viện, độc lập với các biến nhiễu. Cứ tăng mỗi $1 \mathrm{mmol} / \mathrm{L}$ glucose làm tăng odds tử vong 26\% (có ý nghĩa thống kê).

Theo Li Dong-bao (2011), trong các phân tích hồi quy logistic, các yếu tố tiên lượng độc lập liên quan đến tử vong nội viện ở bệnh nhân NMCT cấp là tuổi, glucose máu nhập viện, độ Killip, nhịp tim và loạn nhịp [5]. Với Mikhail K.(2005), sự khác biệt của nguy cơ tử vong liên quan đến glucose máu giữa những bệnh nhân có hoặc không có ĐTĐ vẫn còn khi các phân tích này được thực hiện với biến glucose máu nhập viện là biến liên tục (với mỗi $10 \mathrm{mg} / \mathrm{dL}$ tăng của glucose máu) [9]. Theo Masaharu Ishihara (2012), cứ tăng $1 \mathrm{mmol} / \mathrm{L}$ glucose máu nhập viện là tăng tỉ lệ tử vong trong viện lên $12 \%$ trong phân tích đơn biến và $10 \%$ trong phân tích đa biến [8]. Do nghiên cứu của chúng tôi có cỡ mẫu hạn chế nên kết quả chưa có giá trị thống kể ở nhóm có glucose máu vừa, hơn nữa việc điều chỉnh các biến nhiễu chưa thực sự tốt một phần cũng do hạn chế về cỡ mẫu.

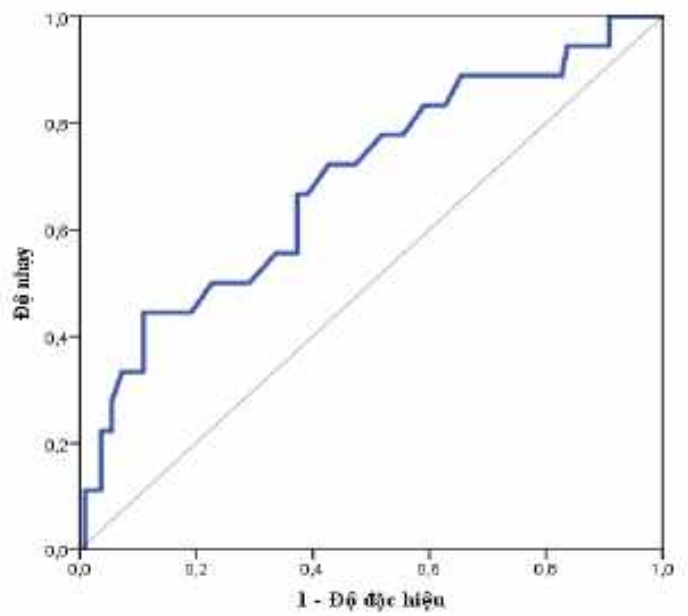

Hình 6. Đường cong ROC của glucose nhập viện với tử vong trong viện 
Khi đánh giá tỉ lệ tử vong dựa vào nồng độ glucose máu lúc nhập viện ta vẽ được đường cong $\mathrm{ROC}$ với $\mathrm{AUC}=0,69$. Giá trị này có ý nghĩa thống kê $(95 \% \mathrm{CI} 0,554-0,829 ; \mathrm{p}<0,05)$. Hơn nữa $\mathrm{AUC}$ được xem là có ý nghĩa lâm sàng khi $\geq 0,7$ nên giá trị $\mathrm{AUC}=0,69$ của nghiên cứu này có thể chấp nhận được. Dựa vào đường cong ROC hoặc chỉ số Youden xác định được điểm tốt nhất có độ nhạy 44,4\% và độ đặc hiệu $89,1 \%$ ứng với điểm cắt của glucose máu lúc nhập viện là $9,6 \mathrm{mmol} / \mathrm{L}$ trong nghiên cứu của chúng tôi. Trong nghiên cứu của Anna T.K. (2012), giá trị điểm cắt tốt nhất của tăng glucose máu do stress ở những bệnh nhân bị NMCT cấp được can thiệp để đánh giá tử vong là $11,28 \mathrm{mmol} / \mathrm{L}$. Giá trị này có độ đặc hiệu và độ nhạy cao nhất trong tiên lượng xấu cho bệnh nhân [10].

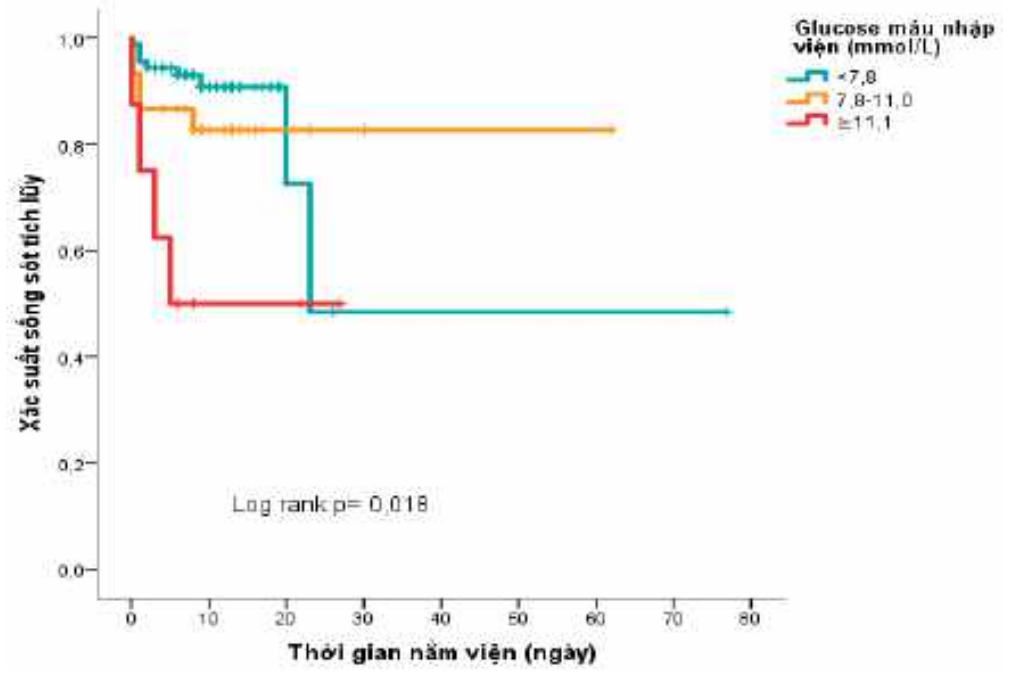

Hình 7. Ước tính Kaplan-Meier về thời gian sống còn của ba nhóm glucose máu nhập viện

Ước tính Kaplan-Meier mô tả xác suất sống còn theo thời gian nằm viện dựa trên dữ liệu của mẫu nghiên cứu. Thời gian từ lúc nằm viện đến khi có biến cố tử vong ở nhóm có glucose máu cao $(\geq 11,1 \mathrm{mmol} / \mathrm{L})$ sớm hơn hai nhóm còn lại. Có sự khác biệt có ý nghĩa thống kê về thời gian sống còn giữa các nhóm glucose máu (test log-rank có $\mathrm{p}=0,018$ ).

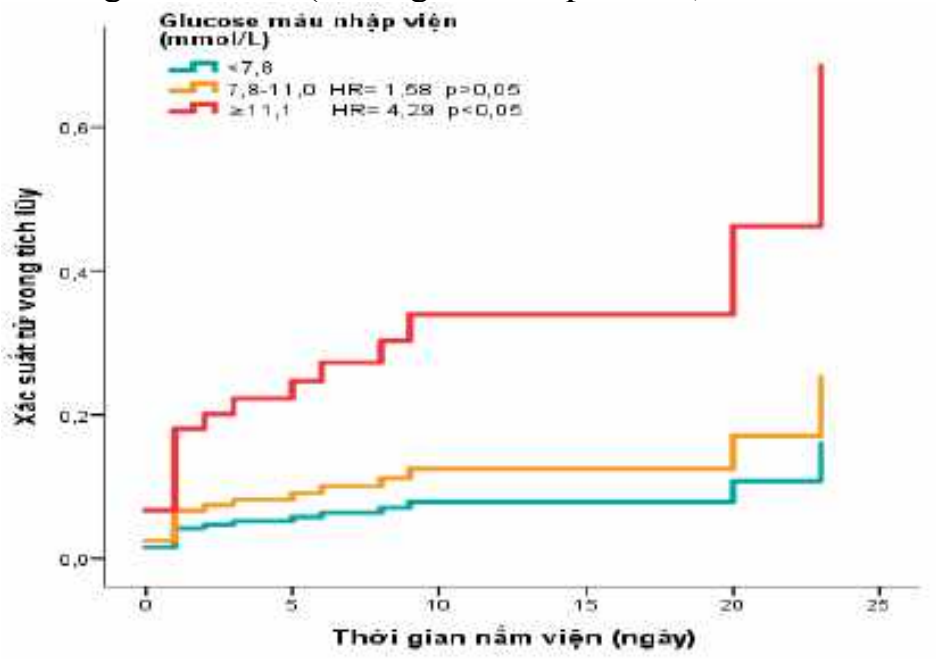

Hình 8. Mô hình hồi qui Cox với ảnh hưởng của glucose máu nhập viện lên tử vong trong viện đã được điều chỉnh 
Theo mô hình hồi qui Cox, sau khi điều chỉnh cho độ tuổi, giới tính, phân độ Killip, sốc tim, so với nhóm glucose $<7,8 \mathrm{mmol} / \mathrm{L}$, nhóm glucose máu cao có tỉ số rủi ro cao gấp 4,29 lần, có ý nghĩa thống kê.

\section{KẾT LUẬN}

Có liên quan glucose máu nhập viện-biến cố tim mạch trong viện. Glucose máu nhập viện là yếu tố tiên lượng độc lập các biến cố xảy ra trong bệnh viện của bệnh nhân nhồi máu cơ tim cấp không đái tháo đường.

\section{TÀI LIỆU THAM KHẢO}

1. Nguyễn Văn Chi (2011), Nghiên cúu đặc điểm lâm sàng, hóa sinh và điều trị tăng đường huyết ở bệnh nhân nhồi máu co tim cấp, Luận án Tiến sĩ Y học, Trường Đại học Y Hà Nội, Hà Nội.

2. Nguyễ் Văn Tân (2015), Nghiên cúu sự khác biệt về lâm sàng, cận lâm sàng và điều trị nhồi máu co tim cấp ở bệnh nhân trên và dưới 65 tuổi, Luận án Tiến sĩ Y học, Đại học Y Dược Thành phố Hồ Chí Minh, Hồ Chí Minh.

3. American Diabetes Association (2018), "Classification and Diagnosis of Diabetes: Standards of Medical Care in Diabetes-2018", Diabetes Care, 41(Suppl 1), pp. S13-S27.

4. Chen P. C., Chua S. K., Hung H. F., et al. (2014), "Admission hyperglycemia predicts poorer short- and long-term outcomes after primary percutaneous coronary intervention for ST-elevation myocardial infarction", J Diabetes Investig, 5(1), pp. 80-6.

5. Dong-bao Li, Qi Hua, Jincheng Guo, et al. (2011), "Admission Glucose Level and In-hospital Outcomes in Diabetic and Non-diabetic Patients with ST-elevation
Acute Myocardial Infarction", Internal Medicine, 50(21), pp. 2471-2475.

6. Dungan Kathleen M., Braithwaite Susan S., Preiser Jean-Charles (2009), "Stress hyperglycaemia", The Lancet, 373(9677), pp. 1798-1807.

7. Ekmekci A., Uluganyan M., Tufan F., et al. (2013), "Impact of admission blood glucose levels on prognosis of elderly patients with ST elevation myocardial infarction treated by primary percutaneous coronary intervention", $J$ Geriatr Cardiol, 10(4), pp. 310-6.

8. Ishihara Masaharu (2012), "Acute Hyperglycemia in Patients With Acute Myocardial Infarction", Circulation Journal, 76(3), pp. 563-571.

9. Kosiborod M., Rathore S. S., Inzucchi S. E., et al. (2005), "Admission glucose and mortality in elderly patients hospitalized with acute myocardial infarction: implications for patients with and without recognized diabetes", Circulation, 111(23), pp. 3078-86.

10. Tomaszuk Kazberuk A., Kozuch M., Malyszko J., et al. (2012), "What level of hyperglycaemia on admission indicates a poor prognosis in patients with myocardial infarction treated invasively?", Kardiol Pol, 70(6), pp. 564-72.

11. Thygesen K., Alpert J. S., Jaffe A. S., et al. (2018), "Fourth universal definition of myocardial infarction (2018)", Eur Heart $J$.

12. Zhao S., Murugiah K., Li N., et al. (2017), "Admission Glucose and Inhospital Mortality after Acute Myocardial Infarction in Patients with or without Diabetes: A Cross-sectional Study", Chin Med J (Engl), 130(7), pp. 767-775. 